\title{
Behavioral control by stimulus components of an imprinting object
}

\author{
LEONARD A. EISERER \\ Keuka College, Keuka Park, New York 14478
}

\begin{abstract}
In the first of three experiments, ducklings that had received prolonged exposure to the visibly moving imprinting object subsequently suppressed ongoing distress vocalization both during brief presentations of the moving object and during brief presentations of its initially neutral stimulus components (i.e., its auditory and static visual features). Only presentations of the moving object were followed by priming aftereffects (namely, enhancement of distress vocalization over a baseline rate). In Experiment 2, weak, but reliable, priming effects were detected after very long presentations of the auditory and static visual features. Experiment 3 found that these features strongly suppressed low, but not high, rates of distress vocalization, while the visibly moving object strongly suppressed both high and low rates. These studies suggest that initially neutral features of an imprinting object acquire the same sort of behavioral control as is exerted by the object when it is in motion, but that this control is somewhat weaker.
\end{abstract}

When a moving object is initially presented to newly hatched ducklings that are emitting distress calls, those calls are almost totally suppressed (Hoffman, Stratton, Newby, \& Barrett, 1970). This suppression, which is generally considered a basic component of the filial response (Sluckin, 1965), is so immediate as to suggest that it represents an unlearned reaction to some aspect of the stimulation provided by the object. Based on a number of findings, it is now apparent that the stimulation provided by the motion of the object is critical for the occurrence of this response. Thus, suppression does not occur when newly hatched ducklings are first exposed to sounds of the object without also seeing it move, nor does it occur if the object remains stationary and silent during its initial presentation (Hoffman et al., 1970).

In short, with the kinds of arbitrarily selected stimuli used in many imprinting experiments with ducklings, the static visual features of the imprinting object (e.g., its specific color, shape, and size, as represented by the object remaining stationary during its presentation) as well as its specific auditory features (i.e., the sound of the moving but visually hidden object) are essentially neutral in their initial effects upon ongoing distress vocalization. This does not preclude the possibility that, for other species of precocial birds, other stimulation besides visual movement might be sufficient to elicit filial

This research was supported by Grant MH-19715 from the National Institute of Mental Health, directed by Dr. Howard S. Hoffman of Bryn Mawr College. The author is no longer affiliated with Keuka College. Current address: 315 Main Street, Penn Yan, New York 14527. responses. Moreover, it is possible that, with ducklings in a natural setting and with a natural mother, other sorts of stimulation besides visual motion (e.g., the mother's specific calls) might also innately elicit filial behavior. But in the laboratory setting, these species-specific signals are not ordinarily components of the arbitrarily selected imprinting objects that are typically used (some laboratory workers employ "natural" imprinting objects, but these are in the minority).

Although the static visual features and the auditory features of a laboratory imprinting object may not innately elicit filial behavior, these features can gradually acquire strong suppressive properties if the ducklings receive prolonged exposure to the object in motion (Eiserer \& Hoffman, 1974; Eiserer, Hoffman, \& Klein, 1975; Hoffman, Eiserer, \& Singer, 1972). Thus, in ducklings that periodically see the imprinting object in motion, the static visual features and the auditory features gradually acquire the ability to suppress distress calls by themselves (i.e., without the accompaniment of visual motion). Importantly, prolonged exposure to these initially neutral features by themselves does not seem sufficient for the acquisition of suppressive properties; repeated exposure to the object in motion is needed (Eiserer \& Hoffman, 1974; Hoffman et al., 1972).

Since the neutral features of a moving imprinting object can acquire the suppressive properties of that object, it might be expected that those features would also acquire any other properties that the moving object may possess. This, however, does not seem to be the case. In addition to suppressive properties, for instance, the moving object also displays "priming" properties in that a brief response-independent 
presentation of the moving object is followed by an enhancement of certain behaviors in imprinted ducklings (Eiserer \& Hoffman, 1973). For example, brief priming presentations of the moving object increase the tendency of ducklings to emit an operant (e.g., pecking a pole) that has previously been reinforced by object presentations. Similarly, certain unlearned behaviors of ducklings (e.g., distress vocalization) are enhanced following presentation of the moving object, relative to the rate of distress vocalization that would have occurred had the object not been presented and withdrawn.

Unlike suppressive properties, however, priming properties do not appear to ever be acquired by the neutral features of the imprinting object despite prolonged exposure to the object in motion. Eiserer and Hoffman (1973), for example, found that under conditions in which presentation of the visual features of the imprinting object almost completely suppressed ongoing distress calls, such presentations were not followed by any observable priming effects. The present series of experiments was directed at obtaining an answer to why the imprinting object's neutral features should apparently acquire strong suppressive properties but no priming properties.

\section{GENERAL METHOD}

\section{Subjects}

Each of the experiments in the present series used a new group of Khaki Campbell ducklings (Anas platyrhynchos domesticus) that were hatched in visual isolation from eggs obtained from George F. Shaw, Inc., West Chester, Pennsylvania. Except for periods spent in the experimental apparatus, each duckling was maintained in an individual housing unit consisting of a 15-gal, white translucent container that was lined with a clear polyethylene bag and partially filled with bedding material. Under these circumstances, the ducklings could hear each other but their visual environment was restricted to that provided by the inside of their individual housing units. While in those units, the ducklings had continuous access to food and water.

\begin{abstract}
Apparatus
The experimental apparatus is illustrated in Figure 1. It consisted of a plywood box $(122 \times 76 \times 76 \mathrm{~cm})$ divided lengthwise by a fine-mesh stainless steel screen into approximately equal compartments, one for the duckling and the other for the imprinting object. Except for special procedures described in Experiment 3, lighting in the subject compartment was provided by two continuously illuminated $75-W$ incandescent lamps mounted above the screen. These lamps were positioned so that unless the stimulus compartment was also illuminated, the light which reflected from the stainless steel screen prevented the subject from seeing into the stimulus compartment.
\end{abstract}

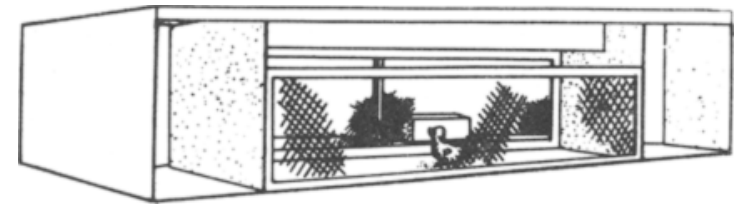

Figure 1. Apparatus used for imprinting and subsequent test procedures.
The imprinting object consisted of a white rectangular foamrubber covering $(22 \times 10 \times 10 \mathrm{~cm})$ mounted over a model train engine which ran the length of the stimulus compartment on H0-ga track. Presentations of the visible moving object were produced by illuminating two overhead $75-\mathrm{W}$ incandescent lamps in its compartment and moving the object back and forth along its track at approximately $30 \mathrm{~cm} / \mathrm{sec}$. Presentations of the static visual features of the imprinting object were produced by illuminating the two stimulus compartment lamps and withholding power from the engine that carried the imprinting object. Presentations of only the auditory features of the imprinting object were produced by moving the object back and forth along its track without illuminating the lamps in the stimulus compartment; the sound thus produced was a broad-band low-frequency noise with an average intensity of $67 \mathrm{~dB}$ re $0.0002 \mathrm{dyne} / \mathrm{cm}^{2}$. Complete stimulus withdrawal was accomplished by extinguishing the compartment lamps and/or stopping the movement of the object.

Distress vocalization of the ducklings was monitored with a specially constructed voice key which, through selective filtering, was sensitive only to those sounds which fall in the frequency range characteristic of distress calls (approximately 3,000$4,000 \mathrm{~Hz}$ ).

\section{EXPERIMENT 1}

Experiment 1 sought to replicate the earlier finding (Eiserer \& Hoffman, 1973) that the static visual features of an imprinting object could acquire strong suppressive properties while not displaying any priming properties. At the same time, the experiment assessed the generality of this effect regarding other initially neutral aspects of the imprinting object-namely, its auditory features. Thus the work was expected to reveal whether the discrepancy between suppressive and priming properties is specific to the visual dimension of an imprinting object, or whether the discrepancy characterizes the object's nonvisual features as well.

In doing so, the present study employed basically the same procedure previously used to assess priming effects in the context of distress vocalization (Eiserer \& Hoffman, 1973). This procedure seemed particularly advantageous for present purposes, since it permitted simultaneous assessment of both the priming properties and the suppressive properties of the test stimuli.

\section{Method}

Subjects. Nine newly hatched Khaki Campbell ducklings were used.

Procedure. During the first 3 days posthatch, each duckling received six exposure sessions with the moving object, at the rate of 2 sessions/day. These sessions consisted of 20 -min periods in the experimental apparatus, during which the moving object was presented continuously. Previous work has indicated that this amount of exposure to the moving object is sufficient for the acquisition of strong suppressive properties by the object's neutral features.

Beginning on Day 4 posthatch, each duckling received one experimental session on each of 3 successive days. In these sessions, the subject was placed in the apparatus with the imprinting object withdrawn. Then, whenever the duckling failed to emit a distress call for $10 \mathrm{sec}$, one of four events occurred: (1) the moving object was presented for $10 \mathrm{sec}$, (2) the visual features were presented for $10 \mathrm{sec}$, (3) the auditory features were 
presented for $10 \mathrm{sec}$, or (4) no stimulus event occurred, but a baseline interval of $10 \mathrm{sec}$ was marked on the operations recorder. After termination of a stimulus event or after $10 \mathrm{sec}$ of the baseline condition had elapsed, no further event occurred until the duckling again went $10 \mathrm{sec}$ without a distress call. Each session ended when two trials of each type, occurring in random order, had been completed.

\section{Results}

Figure 2 shows the mean number of seconds of distress vocalization emitted during, and in the $10 \mathrm{sec}$ immediately following, presentations of the moving object and its visual and auditory features, relative to parallel baseline periods. As can be seen, distress vocalization was suppressed during presentations of all these stimuli relative to baseline (Graph $A$ in the figure); however, an enhancement of distress calls over baseline occurred only after presentation of the moving object (Graph B in the figure).

For statistical analysis of the data, the two sections of Figure 2 were handled separately. Regarding the distress vocalization emitted during stimulus presentations, an analysis of variance for repeated measures indicated significant treatment effects $(F=10.7$, df $=3 / 24, p<.05)$, and a NewmanKeuls test was then used to assess all possible individual comparisons. This test indicated a significant difference between amount of distress vocalization during the baseline condition and during each of the three stimulus conditions ( $p<.05$, in all cases) and insignificant differences among the stimulus conditions themselves ( $p>.05$, in all cases).

Regarding the distress vocalization emitted during the $10 \mathrm{sec}$ immediately following each of the four events, an analysis of variance yielded significant treatment effects $(F=6.9$, df $=3 / 24, p<.05)$. A Newman-Keuls test then revealed a significant difference between amounts of distress vocalization following offset of the moving object and following each of the other three events ( $p<.05$, in all cases), and insignificant differences among the distress vocalization that followed presentations of the visual and auditory features and the baseline event $(p>.05$, in all cases).

In addition to the above findings, the present study allowed assessment of the persistence of the priming effect by measuring the time interval that occurred between trials. Since each trial began only when the duckling went $10 \mathrm{sec}$ without distress vocalization, the intertrial interval (ITI) was long or short depending on the persistence of the duckling in emitting distress calls. Figure 3 shows the mean ITI (as an index of response perseveration) that followed presentations of the moving object, its visual and auditory features, and the baseline interval. Significant treatment effects were verified by an analysis of variance $(F=4.3$, df $=3 / 24, p<.05)$. A NewmanKeuls test revealed that response perseveration was reliably greater after presentations of the moving ob-

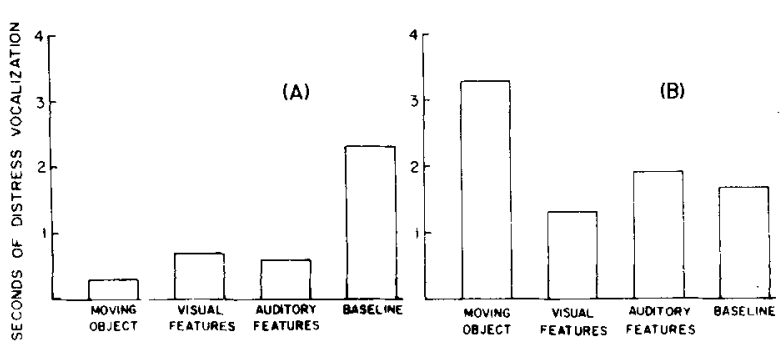

Figure 2. Mean number of seconds of distress vocalization emitted during (Graph A), and in the 10 sec immediately following (Graph B), presentations of the moving object and its visual and auditory features, relative to parallel baseline periods.

ject than after presentations of either the visual or the auditory features or after the baseline event $(p<.05$, in all cases). Response perseveration did not differ significantly on baseline trials and trials with the visual or auditory features ( $p>.05$, in all cases).

To summarize the present results, comparable amounts of distress call suppression were produced by presentations of the moving object and its visual and auditory features. Only presentations of the moving object, however, produced detectable enhancement of subsequent distress vocalization.

\section{Discussion}

Numerous similarities exist between the behavioral control exerted by the static visual features and that exerted by the auditory features of an arbitrarily selected imprinting object. Among these similarities are the facts that both types of features are initially neutral in their effects upon ongoing distress vocalization (Eiserer \& Hoffman, 1974; Hoffman et al., 1972), both types acquire suppressive properties only when they are paired with visual movement (Eiserer \& Hoffman, 1974; Hoffman et al., 1972), and, after such acquisition, both types subsequently retain those suppressive properties throughout prolonged periods in which they are no longer accompanied by visual movement (Eiserer, Hoffman, \& Klein, 1975). The present experiment adds still another

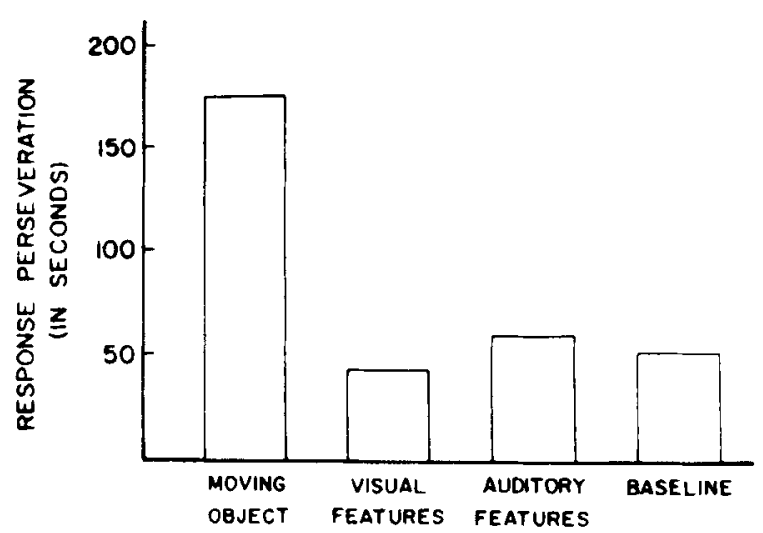

Figure 3. Length of intertrial interval following presentations of the moving object, the visual features, the auditory features, and following the comparable baseline periods. 
similarity, namely, that both types of features appear to lack priming properties, even when their suppressive properties are substantial. Taken together, these many similarities suggest that, with an arbitrarily selected imprinting object, the auditory features are functionally comparable to the static visual features.

Another implication of the present findings is that the lack of correspondence between suppression and priming is not merely idiosyncratic to the visual aspects of an imprinting object, but instead characterizes more generally the object's initially neutral stimulus components. Proceeding from this conclusion, the next two experiments attempted to determine whether differential sensitivity in the measurement of suppression vs. priming might account for the discrepancy existing between the occurrence of these two effects.

\section{EXPERIMENT 2}

Despite the apparent lack of priming properties by both the visual and the auditory features of the imprinting object, the possibility remains that the two types of features would produce priming effects under more conducive testing conditions. For example, since priming effects with the moving object have been found to increase with longer priming presentations (Eiserer \& Hoffman, 1973), it seems possible that very long primes with the visual and the auditory features might produce detectable effects. Experiment 2 explored this possibility by varying prime duration with the visual and the auditory features.

As another effort to optimize testing conditions, Experiment 2 examined priming effects in the context of pole-pecking (i.e., with ducklings who had been trained to peck a pole for brief presentations of the moving object) rather than in the context of distress vocalization. Previous work (Eiserer \& Hoffman, 1973) has suggested that the same mechanisms underlie priming of both responses, since certain variables (e.g., prime duration and priming with the visual features) affect both behaviors in comparable ways. Measurement of the pecking response, however, offered an advantage over measurement of distress calls in that peking can be maintained over a large number of days (e.g., see Hoffman \& Kozma, 1967), whereas ducklings frequently cease emitting baseline distress vocalization once they have habituated to the experimental apparatus-a process that may take but a few hours of exposure to the apparatus (Eiserer et al., 1975). Thus more trials were potentially possible with polepecking than with distress vocalization, and more trials would permit more sensitive assessment of any priming effects that might occur.

\section{Method}

Subjects. Six newly hatched Khaki Campbell ducklings were used.

Apparatus. In the present experiment, the apparatus was modified by mounting a balsa-wood pole $(1 \times 1 \times 30 \mathrm{~cm})$ on the wall above the center of the screen so that it hung down vertically to the floor of the subject compartment. Pecking responses on this pole, which was attached to a pigeon key, initiated presentation of the moving object for a duration determined by the setting of a timer. The peck force required to activate the object was approximately $2 \mathrm{~g}$.

Procedure. Each duckling was exposed to the continuously moving object in two 20 -min imprinting sessions per day for the first 3 days posthatch. Then, beginning on Day 4 , each subject was trained to peck the pole with presentation of the moving object as reinforcement. During these procedures, the experimenter observed the duckling via closed-circuit television and presented the object very briefly (approximately $.5 \mathrm{sec}$ ) when the bird approached the pole. Once the duckling was in the vicinity of the pole, only motions which more and more closely resembled a pole peck were reinforced until the duckling actually pecked the pole.

The first peck produced the moving object for $5 \mathrm{sec}$, but as the response became stronger, this duration was increased to $15 \mathrm{sec}$. Since the timer controlling presentation of the object did not reset with additional responses after the one that initiated the 15-sec presentation, time for object presence did not accumulate. As a result, the imprinting object could not be kept continuously present.

After the pecking response was firmly established, the ducklings were randomly divided into two groups of three subjects each. For Group 1, 15-sec presentations of the moving object were made contingent upon pole pecks in each of 12 experimental sessions. Six of these sessions were designed to assess priming effects by the moving object, while the other six sessions were used to assess priming effects by the object's visual features. The two types of sessions occurred in random sequence.

During moving-object sessions, one of six possible events occurred whenever the duckling permitted 5 min to pass without a response; either the moving object was presented for 3,30 , or $180 \mathrm{sec}$ (prime trials) or it was not presented but periods of 3, 30, or $180 \mathrm{sec}$ were designated on the operations recorder so that the duration of these baseline events could be respectively determined. Following a given event, a 5 -min no-response criterion had again to be met before the next trial occurred.

These same basic contingencies were in effect during visualfeature sessions except that prime trials consisted of experimenterinitiated presentations of the visual features rather than of the moving object. One session was run per day, and each session consisted of two trials of each type (i.e., the three different durations of prime and baseline trials).

The three ducklings in Group 2 were also run in 12 experimental sessions at the rate of $1 /$ day. The design of these sessions was in every way identical to that described above, except that instead of visual-feature sessions, the ducklings in Group 2 received auditory-feature sessions. During these sessions, experimenterinitiated presentations of the auditory features occurred on prime trials.

\section{Results}

Priming effects were assessed in terms of the probability of response initiation in the 1-min interval immediately following termination of priming presentations relative to the comparable response probability that followed baseline events. The means of these probabilities, which represent the proportion of the 1-min measurement periods that contained one or 
more responses, are presented in Figure 4 for both Group 1 (top two graphs) and Group 2 (bottom two graphs). For both groups of subjects, the probabilities for all three baseline intervals were averaged together, since the appropriate analyses of variance indicated no significant differences among them. During visual-feature sessions for Group $1, \bar{X}=.15$ for 3-sec baseline, .10 for $30-\mathrm{sec}$ baseline, and .09 for 180-sec baseline; during moving-object sessions, $\overline{\mathrm{X}}=.05$ for 3 -sec baseline, .13 for 30 -sec baseline, and .08 for 180 -sec baseline. During auditory-feature sessions for Group 2, $\bar{X}=.20$ for $3-\mathrm{sec}$ baseline, .29 for 30 -sec baseline, and .26 for 180 -sec baseline; during moving-object sessions, $\bar{X}=.10$ for $3-\mathrm{sec}$ baseline, .19 for $30-\mathrm{sec}$ baseline, and .26 for $180-\mathrm{sec}$ baseline.

As can be seen from the data of Group 1 in Figure 4 , response probability following presentations for both the visual features and the moving object increased as a direct function of the duration of the priming presentation. These increases, however, were much greater with primes by the moving object than with primes by the visual features. The reliability of these trends was verified by an analysis of variance, which yielded a significant effect of prime duration $(F=10.0, \mathrm{df}=3 / 6, \mathrm{p}<.05)$, a significant interaction between the effects of stimulus and prime duration $(F=6.9, \mathrm{df}=3 / 6, \mathrm{p}<.05)$, and a nonsignificant stimulus effect $(\mathrm{F}=1.4$, df $=1 / 2, p>.05$ ).

Comparable effects are depicted in the data of Group 2 in Figure 4, since response probability following presentations of both the auditory features and the moving object increased directly as a function of duration of the priming presentation. These increases, particularly at the 30 -sec duration, appeared to be greater with primes by the moving object than with primes by the auditory features. The reliability of these trends was confirmed by an analysis of variance which yielded a significant effect of prime durations $(F=18.8$, df $=3 / 6, p<.05)$, a significant interaction between the effects of stimulus and prime duration $(F=5.7$, df $=3 / 6$, $\mathrm{p}<.05$ ), and a nonsignificant stimulus effect $(F=3.7, \mathrm{df}=1 / 2, \mathrm{p}>.05)$.

\section{Discussion}

As can be seen in Figure 4, Groups 1 and 2 were not equivalent in their responses to primes by the moving object; indeed, across all three prime durations, Group 2 displayed considerably higher response probabilities than did Group 1. This intergroup discrepancy, which probably reflected mere chance individual differences among the subjects (who numbered only three in each group), causes problems in interpreting the greater response level that occurred with respect to the auditory features compared to the visual features. Thus, rather than
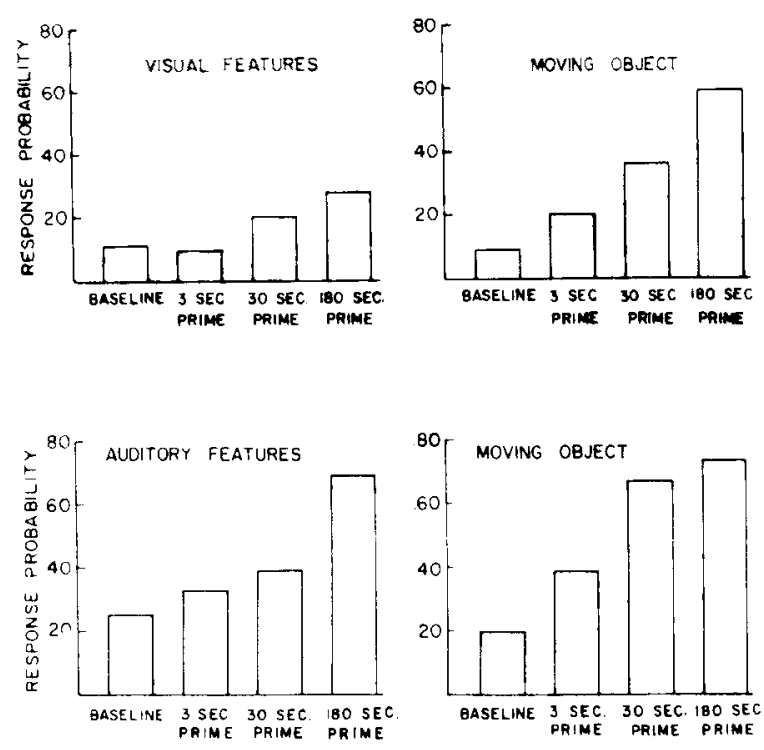

Figure 4. Top two graphs: Response probability for ducklings in Group 1 during the 1 -min interval immediately following short and long primes with the visual features and the moving object, and following the comparable baseline periods. Bottom two graphs: Response probability for ducklings in Group 2 during the 1-min interval immediately following short and long primes with the auditory features and the moving object, and following the comparable baseline periods.

reflecting a true distinction between sensory modalities, the auditory-visual difference could simply reflect the generally higher response level that Group 2 generated compared to Group 1. Nevertheless, the crucial finding of the present experiment is that response probability increased directly as a function of prime duration regardless of whether the prime involved the moving object, its visual features, or its auditory features, even though such increases tended to be greatest with the moving object.

As noted by Eiserer and Hoffman (1973), the finding of greater priming effects after long presentations of the imprinting object implies that priming effects do not derive solely from events associated with either stimulus onset or stimulus offset, but instead reflect events that occur and in some sense cumulate during stimulus presence. In this context, it is of interest that an increase in priming effects as a direct function of magnitude of the priming stimulus has repeatedly been found with positively reinforcing brain stimulation. Gallistel (1966), for example, found that pretrial brain stimulation increased runway speed if trains of six pulses of stimulation were delivered but not if only three pulses were given. Similary, Howarth and Deutsch (1962) reported that the aftereffects of brain stimulation, as measured by resistance to subsequent extinction of a leverpress response, increased with increases in prime intensity and duration. At the neural level, Rolls (1971) found that aftereffects of brain stimulation delivered in the median forebrain bundle, measured in terms of single 
unit firing in the brainstem arousal system and in terms of electroencephalogram desynchronization, showed both greater magnitude and longer persistence as number of brain stimulation trains increased from 1 to 20.

Although priming effects with both brain stimulation and an imprinting stimulus vary directly with prime magnitude, this functional relation does not appear to hold true for priming effects with water and food. Bruce (1937) found an increase in the runway speed of thirsty rats after administering small amounts of pretrial water, but found a decrease in speed after large amounts of pretrial water. A comparable result with food reinforcement was reported by Morgan and Fields (1938). Of course, it is very likely that processes of satiety-which appear to be of minimal importance with brain stimulation or stimulation from an imprinting object-served to counteract any priming effects that might otherwise have been induced by large amounts of food or water.

In any event, the present experiment-by yielding reliable priming effects by both the visual and the auditory features - has helped to resolve the apparent discrepancy between the suppressive properties and the priming properties of stimulus components of an imprinting object. It is now clear that this discrepancy reflects at most only quantitative differences inasmuch as priming effects are weak and suppressive effects appear strong with stimulus components of an imprinting object. Whether the suppression of distress calls engendered by these stimulus components is really as powerful as it has appeared in past studies (i.e., as powerful as suppression produced by the moving object) was investigated in Experiment 3 .

\section{EXPERIMENT 3}

Any difference that may exist between the suppressive properties of the moving object and the suppressive properties of its visual and auditory features may have gone undetected in earlier work because of ceiling effects in measuring suppression. After ducklings have received prolonged exposure to the moving object, for example, the visual features typically reduce ongoing distress calls to a point near zero (e.g., Eiserer \& Hoffman, 1973). Although such complete suppression might suggest that the visual features are more or less equivalent to the moving object in suppressive properties, it is also possible that relatively little suppressive strength is necessary for complete reduction of ongoing distress vocalization. If so, then the moving object could conceivably have much stronger suppressive properties than its visual features even though the latter has sufficient strength to cause nearly complete termination of distress vocalization.
To test this possibility, the present experiment attempted to induce an unusually high baseline rate of distress vocalization in order to more severely tax the suppressive control of the moving object, its visual features, and its auditory features. It was anticipated that under these conditions, suppression by all of the stimuli would weaken; however, a greater weakening by the visual and the auditory features (relative to the moving object itself) should occur if, in fact, the suppressive properties of those stimuli are not as strong as the suppressive properties of the moving object.

\section{Method}

Subjects. Eight newly hatched Khaki Campbell ducklings were used.

Apparatus. For the purposes of the present experiment, the balsa-wood pole was removed from the imprinting chamber.

Procedure. During the first 3 days posthatch, each duckling received six 20-min imprinting sessions at the rate of 2 sessions/ day. In these sessions, the imprinting object moved continuously along its track in the illuminated stimulus compartment, and the houselights in the subject compartment were maintained at relatively dim illumination (the photometric brightness of the finemesh screen was approximately $.08 \mathrm{fL}$ during complete stimulus withdrawal). On Day 4 posthatch, the ducklings were randomly divided into two groups of four subjects. The remaining experimental procedures were completed on this day.

Ducklings in Group 1 received two separate distress-call tests. Both tests consisted of three 20-sec presentations of the moving object occurring in random sequence with three 20 -sec presentations of the visual features; each of these stimulus presentations was preceded by a 20 -sec period of complete stimulus withdrawal. During one of the tests, the houselights remained dim (approximately $.08 \mathrm{fL}$ ), and during the other test, the houselights were brightened (approximately $1.5 \mathrm{fL}$ ). Sequence of lighting conditions was counterbalanced across subjects.

Nearly identical procedures were employed in testing ducklings in Group 2, except that presentations of the auditory features occurred during periods in which the visual features had been presented to Group 1.

\section{Results}

Figure 5 shows the average number of seconds of distress vocalization that both groups of ducklings emitted during periods of stimulus presentation and withdrawal under normal (i.e., dim) and bright illumination. Inspection of the data for Group 1 (top two graphs) reveals that under the normally dim illumination, presentations of the visual features and of the moving object almost completely suppressed baseline distress vocalization. Under bright illumination, however, baseline distress vocalization increased and the visual features weakened in their suppressive control while the moving object remained strong. Similar effects were shown by Group 2 (bottom two graphs), in that presentations of both the auditory features and the moving object reduced baseline distress vocalization to near zero under conditions of normal illumination, but only presentations of the moving object still produced nearly complete suppression under bright illumination.

The reliability of all of these trends was supported 

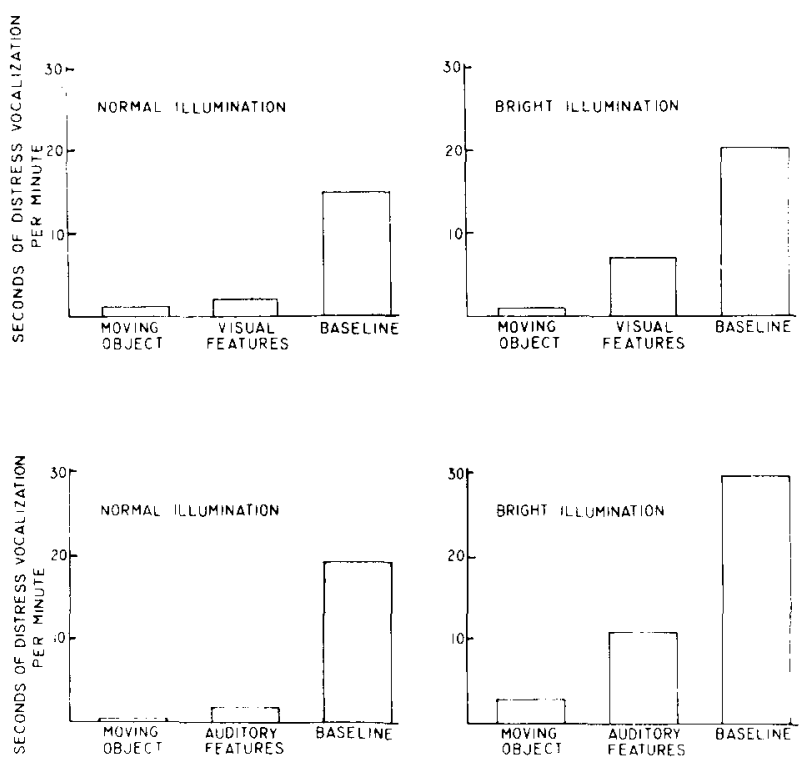

Figure 5. Top two graphs: Rate of distress vocalization emitted by ducklings in Group 1 during the presentations of the moving object, the visual features, and during the baseline periods, under both normal and bright illumination. Bottom two graphs: Rate of distress vocalization emitted by ducklings in Group 2 during presentations of the moving object, the auditory features, and during the baseline periods, under both normal and bright illumination.

by statistical analyses. For the data of Group 1, an analysis of variance yielded a significant effect of lighting condition $(F=12.1, \mathrm{df}=1 / 3, p<.05)$, a significant effect of stimulus condition $(F=22.3$, $\mathrm{df}=2 / 6, \mathrm{p}<.05)$, and a significant interaction between the effects of lighting and stimulus condition $(\mathrm{F}=5.7, \mathrm{df}=2 / 6, \mathrm{p}<.05)$. $\mathrm{F}$ tests for simple effects then revealed that, while amount of distress vocalization during presentations of the moving object did not change significantly as a function of illumination $(F=0.0, \mathrm{df}=1 / 6, \mathrm{p}>.05)$, amount of distress vocalization during presentations of the visual features was greater under bright illumination than under dim illumination $(F=17.2, \mathrm{df}=1 / 6$, $\mathrm{p}<.05$ ). The amount of baseline distress vocalization was also significantly greater under bright illumination than under dim illumination $(F=17.2$, $\mathrm{df}=1 / 6, \mathrm{p}<.05$ )

For the data of Group 2, an analysis of variance indicated a significant effect of lighting condition $(F=23.1, \mathrm{df}=1 / 3, \mathrm{p}<.05)$, a significant effect of stimulus condition $(F=28.8, \mathrm{df}=2 / 6, \mathrm{p}<.05)$, and a significant interaction between the effects of lighting and stimulus condition $(F=5.5, \mathrm{df}=2 / 6$, $p<.05)$. F tests for simple effects verified that, while amount of distress vocalization during presentations of the moving object did not change significantly as a function of illumination $(F=0.14, \mathrm{df}=$ $1 / 6, p>.05)$, amount of distress vocalization during presentations of the auditory features was reliably greater under bright illumination than under dim illumination $(F=20.1$, df $=1 / 6, p<.05)$. The amount of baseline distress vocalization was also significantly greater under bright illumination than under dim illumination $(F=20.3, \mathrm{df}=1 / 6$, $\mathrm{p}<.05)$.

\section{Discussion}

The present study found that both the visual and auditory features of the imprinting object almost completely suppressed ongoing distress vocalization when the houselights were dimly illuminated, but that these features only partially suppressed distress vocalization when the houselights were brightly illuminated. In contrast, the moving object exerted nearly complete suppression under both dim and bright houselights. These results suggest that the suppressive properties of the moving object were stronger than the suppressive properties of the object's visual and auditory features.

The increase in baseline rate of distress vocalization that both groups of ducklings showed under bright illumination may have represented an aversive reaction to the bright lights themselves. Alternatively, the subjects may have been reacting to the elements of novelty and strangeness that the brighter illumination introduced into the ducklings' environment. Very probably, stimulus aspects of the experimental chamber that had previously been either poorly perceived or unseen altogether emerged into sudden conspicuous under the bright illumination-reason enough for 4-day-old ducklings to become more distressful than usual. In any event, as is obvious from the results of the present experiment, the observed increase in baseline distress vocalization was sufficient to prevent a ceiling effect from masking reliable differences in the strength of suppression produced by the moving object relative to the suppression produced by its visual and auditory features.

\section{CONCLUSIONS}

The present work has resolved the discrepancy between the apparently strong suppressive properties and the apparently absent priming properties of an imprinting object's initially neutral visual and auditory features. The suppressive control that these features acquire had in previous research (and also in Experiment 1) appeared to be just as powerful as the control exerted by the moving object itself, but was found in Experiment 3 to be, in fact, weaker. Similarly, the priming effects engendered by presenting the stimulus components of an imprinting object, rather than being absent (as found in previous work, and again in Experiment 1), were demonstrated in Experiment 2 to be merely weaker than those engendered by presenting the moving object. In short, the present series of experiments reveals that after 
ducklings have received prolonged exposure to the imprinting object in motion, the object's initially neutral stimulus components exhibit the same sort of behavioral control that is exerted by the object when it is in motion, but this control is weaker.

In assessing the implications of this conclusion, it is worthwhile to consider possible alternative results that the present work might have produced. The visual and auditory features, for example, might have failed to generate observable priming effects despite increases in duration of priming presentations. And with increases in baseline distress vocalization, suppression by the moving object might have weakened as much as suppression by its stimulus components. Such results would have strengthened the appearance of qualitative discrepancy between the suppressive and priming properties of the intially neutral features of an imprinting object, and would accordingly have suggested that suppression and priming are mediated by two different and relatively independent mechanisms. Instead, the results of the present work reveal that suppression and priming effects covary (since stimuli that suppress weakly also prime weakly), implying that their underlying mechanisms may be closely related.

\section{REFERENCES}

BRUCE, R. H. An experimental investigation of the thirst drive in rats with especial reference to the goal-gradient hypothesis. Journal of General Psychology, 1937, 17, 49-60.
Eiserer, L. A., \& Hoffman, H. S. Priming of ducklings' responses by presenting an imprinted stimulus. Journal of Comparative and Physiological Psychology, 1973, 82, 345-359.

Eiserer, L. A., \& Hoffman, H. S. Acquisition of behavioral control by the auditory features of an imprinting object. Animal Learning \& Behavior, 1974, 2, 275-277.

Eiserer, L. A., Hoffman, H. S., \& Klein, S. H. Persistence of acquired behavioral control in the context of imprinting. Journal of the Experimental Analysis of Behavior, 1975, 24, 255-266.

Gallistel, C. R. Motivating effects in self-stimulation. Journal of Comparative and Physiological Psychology, 1966, 62, 95-101.

Hoffman, H. S., Eiserer, L. A., \& Singer, D. Acquisition of behavioral control by a stationary imprinting stimulus. Psychonomic Science, 1972, 26, 146-148.

Hoffman, H. S., \& Kozma, F., JR. Behavioral control by an imprinted stimulus: Long term effects. Journal of the Experimental A nalysis of Behavior, 1967, 10, 495-501.

Hoffman, H. S., Stratton, J. W., Newby, V., \& Barrett, J. E. Development of behavioral control by an inprinting stimulus. Journal of Comparative and Physiological Psychology, 1970, 71, 229-236.

Howarth, C. I., \& Deutsch, J. A. Drive decay: The cause of fast extinction of habits learned for brain stimulation. Science, 1962 , 137, 35-36.

Morgan, C. T., \& Fields, P. E. The effect of variable preliminary feeding upon the rat's speed-of-locomotion. Joumal of Comparative Psychology, 1938, 26, 331-348.

RoLLs, E. T. Involvement of brainstem units in medial forebrain bundle self-stimulation. Physiology and Behavior, 1971, 7, 297-310.

SluckIN, W. Imprinting and early learning. Chicago: Aldine, 1965.

(Received for publication August 27, 1976; revision accepted January $11,1977$. 